\title{
Improving Airfoil Lift Prediction
}

\author{
Giridhar Ramanujam* and Hüseyin Özdemir ${ }^{\dagger}$ \\ Energy Research Centre of the Netherlands(ECN), 1755LE, Petten, The Netherlands
}

\begin{abstract}
An improved formulation for lift estimation for integral boundary layer (IBL) methods (i.e. RFOIL, XFOIL) for thick airfoils is presented. Lift over-prediction ( $5-10 \%$ around $(l / d)_{\max }$ ) in RFOIL (similarly in XFOIL) is observed for thick airfoils for a wide range of angles of attack. The lift slope is over-predicted resulting in the increasing error in lift with increasing angle of attack. The wake geometry in RFOIL and XFOIL is determined from the inviscid calculations which seems to give rise to the above problem. A scheme has been developed for IBL methods and implemented in RFOIL, to include the effects of viscous flow on the wake geometry which lead to improved lift prediction for thick airfoils. New insights were obtained regarding the discrepancy in predicting the maximum lift. It is observed that lift over-prediction persists in case of thick trailing edge (TTE) airfoils even with the improved method. The cause for this behavior is identified and discussed in order to evaluate the possibilities of improvement. High Reynolds number and increased free-stream turbulence intensity have been observed to inhibit the onset of flow separation and delay stall. These effects are not yet captured accurately in RFOIL indicating a need for further investigation.
\end{abstract}

\section{Nomenclature}

$\begin{array}{ll}\alpha & \text { Angle of attack } \\ c & \text { Airfoil chord length } \\ c_{d} & \text { Sectional drag coefficient } \\ c_{l} & \text { Sectional lift coefficient } \\ h_{T E} & \text { Airfoil trailing edge thickness } \\ M_{\infty} & \text { Free stream Mach number } \\ N_{c r i t} & \text { Critical amplification factor } \\ R e & \text { Chord length based Reynolds number } \\ X, Y & \text { Cartesian space coordinates }\end{array}$

\section{Introduction}

Accurate prediction of aerodynamic characteristics of wind turbine blade sections (airfoils) is essential for predictions of loads on the blades and power performance evaluation. Predominantly, low fidelity CFD methods such as panel methods and vortex lattice methods with a viscous-inviscid interaction scheme are employed for this purpose. Thick airfoils are commonly used in wind turbine blades with the thickness varying from $15 \%$ of chord at the tip section to about $50 \%$ of chord at the root section. It is thus important to have an accurate prediction for lift in order to determine the loads on the wind turbine blade. Additionally, lift has a larger contribution to the rotor torque and thus, accurate prediction of lift will result in accurate prediction of rotor performance. With the trend of size of wind turbines increasing, thicker airfoils are becoming more and more important as the structural requirements need to be satisfied while maintaining optimal aerodynamic performance. Discrepancies in lift and drag prediction in low fidelity CFD methods for thick airfoils has been reported in literature. ${ }^{1,2}$ Under-prediction of drag associated with thick trailing edge (TTE) airfoils and thick wind turbine airfoils is observed in XFOIL and RFOIL predictions. The latter has

*Researcher, Wind Energy Unit, Westerduinweg 3, giridhar.ramanujam@gmail.com. Member AIAA

${ }^{\dagger}$ Researcher, Wind Energy Unit, Westerduinweg 3, h.ozdemir@ecn.nl. Member AIAA. 
already been addressed by the authors in a previous study, ${ }^{2}$ where a semi-empirical correction for drag was formulated. For TTE airfoils, the issue of drag under-prediction is yet to be solved although the location of these airfoils in the root region of the blade implies that lift has more significance on turbine performance than drag. Lift and lift slope over-prediction is another issue that is observed in these methods for thick airfoils as well as TTE airfoils. The maximum lift is also over-predicted indicating a discrepancy in predicting the onset of stall. The present study focuses on identifying the cause of errors in lift prediction in integral boundary layer (IBL) methods like RFOIL and present solutions to improve these predictions.

\section{Aerodynamic Methods}

For the analysis presented in this paper, RFOIL $^{1}$ has been used. RFOIL is an aerodynamic design and analysis method based on XFOIL, ${ }^{3}$ developed by a consortium of ECN, NLR and TU Delft after ECN acquired the XFOIL code. After 1996, ECN maintained and improved the tool. XFOIL ${ }^{\mathrm{a}}$ is a viscous-inviscid interaction (VII) method for predicting flow about airfoils developed by Mark Drela at MIT. It utilizes a linear-vorticity panel method with Karman-Tsien compressibility correction for analysis in direct and mixedinverse modes. Source distributions superimposed on the airfoil and wake permit modelling of viscous layer effects on potential flow results. A two-equation lagged dissipation integral method is used to represent the viscous layers. Both laminar and turbulent flows are treated with an $e^{N}$-type amplification formulation determining the transition point. The boundary layer and transition equations are solved simultaneously with the inviscid flow field by a global Newton method. The procedure is especially suitable for rapid analysis of low Reynolds number flows around airfoils with transitional separation bubbles. RFOIL is a modified version of XFOIL featuring an improved prediction for the maximum lift coefficient and includes a method for predicting the effect of rotation on airfoil characteristics. Regarding the maximum lift in particular, numerical stability improvements were obtained by using the Schlichting velocity profiles for the turbulent boundary layer, instead of Swafford's velocity profiles incorporated in XFOIL. Furthermore, the shear lag coefficient in Green's lag entrainment equation of the turbulent boundary layer model was adjusted based on the shape factor of the boundary layer for deviation from the equilibrium flow observed at high values of the shape factor. Both these methods exhibit a noticeable over-prediction in lift coefficient for all angles of attack with the over-prediction becoming more pronounced as the airfoil becomes thicker and also with increasing angle of attack.

\section{Approach to improve lift prediction}

Before going into the details about the scheme implemented to improve lift prediction, the procedure adopted by RFOIL (or any XFOIL like methods) to calculate the aerodynamic characteristics of an airfoil is explained for the sake of clarity. The flowchart in figure 1 explains briefly how VII methods like RFOIL calculate the aerodynamic characteristics of airfoils.

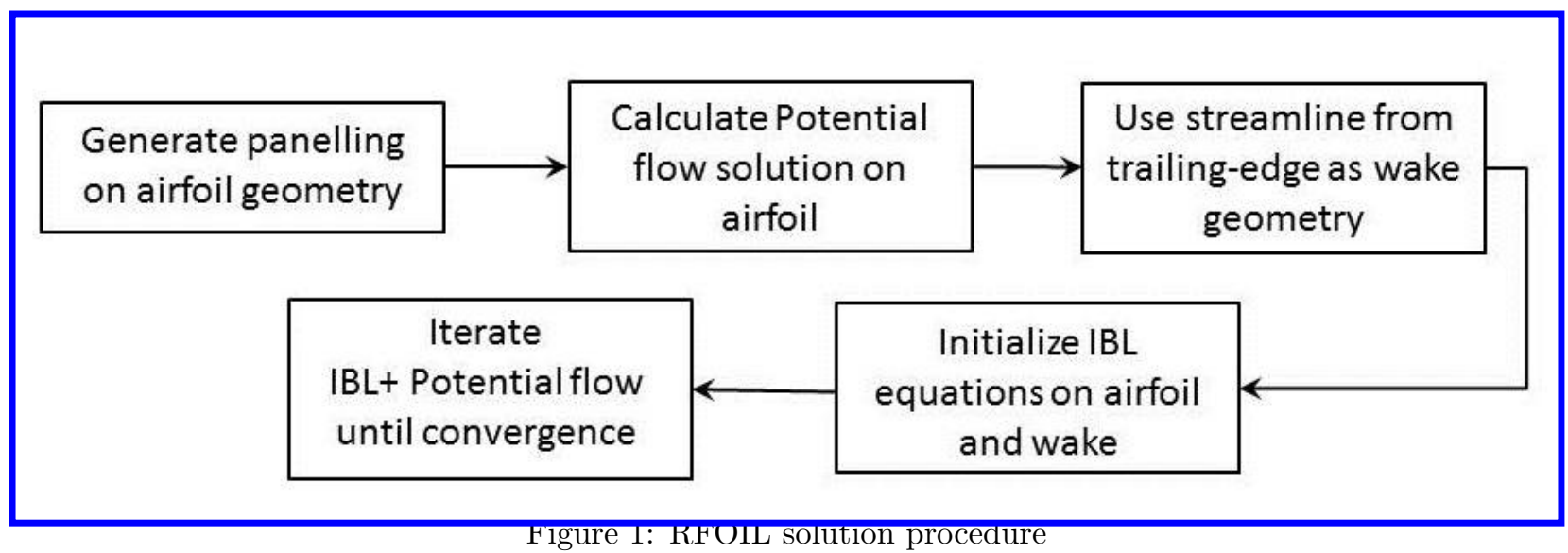

The input to the method is the airfoil coordinates based on which the panelling is generated, subject to

$\overline{\text { a } \quad \text { Refers to XFOIL } 6.99 \text { unless stated otherwise }}$ 
input parameters regulating the panel density and distribution. Once the panelling is complete, an influence coefficient matrix is set up to calculate the influence of all panels on each other. The system of equations thus formed is solved to obtain the strength of the vortex distribution in the panels based on which the inviscid pressure distribution is calculated. To proceed to the viscous calculation, a wake geometry is required in order to set up the direction along which the IBL equations will be solved in the wake of the airfoil. This is done by using the dividing streamline in the wake obtained from the inviscid calculation as the wake geometry for viscous calculations. The dividing streamline in the wake is the streamline originating from the trailing edge of the airfoil. Once the airfoil and wake geometry if calculated, the viscous calculations are carried out using a strong interaction scheme. As a result of the viscous calculations, a source distribution is established on each panel in the airfoil and the wake representing the displacement effect of the boundary layer. The lift and moment coefficients are calculated using the effective edge velocity distribution on the airfoil and the drag coefficient is calculated based on the momentum loss thickness at the end of the wake (one chord-length downstream the airfoil).

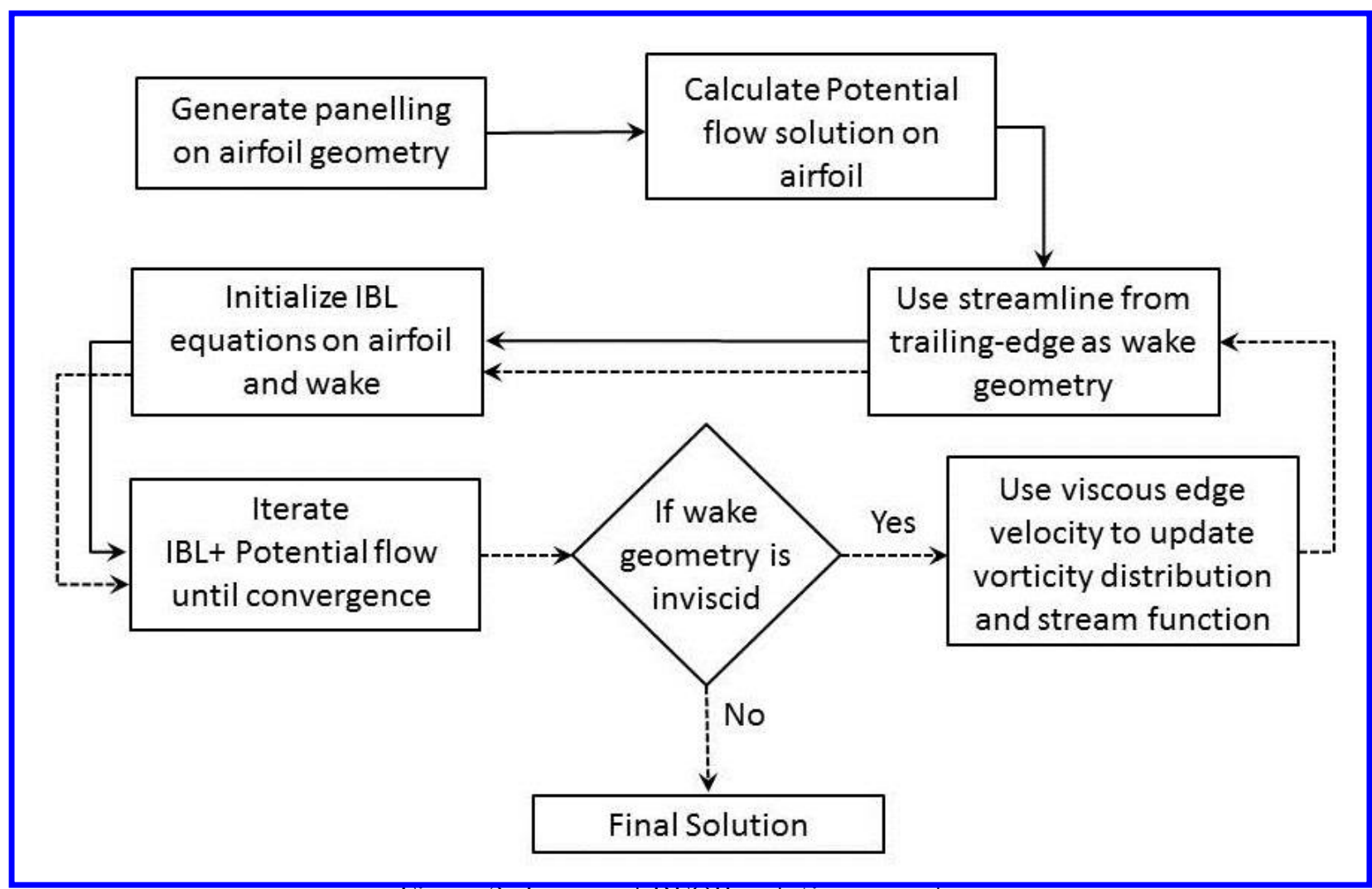

Figure 2: Improved RFOIL solution procedure

In RFOIL (as in XFOIL ${ }^{3}$ ), the wake geometry for the boundary layer calculation in the wake is determined by the inviscid calculation ${ }^{4}$ as decribed in figure 1 . If lift is specified, then the wake trajectory for a viscous calculation is taken from an inviscid solution at the specified lift. If angle of attack is specified, then the wake trajectory is taken from an inviscid solution at that angle of attack. This is not strictly correct, since viscous effects will in general decrease lift and change the trajectory. This effect increases as the angle of attack increases and might result in over-prediction of lift at higher angles, thereby increasing the lift slope. Since the definition of wake geometry has always been a grey area in case of steady state panel and vortex methods, a detailed study on including the viscous effects on the definition of wake geometry might yield some new insight.

In order to include the viscous effects in determining the wake geometry, the procedure described in figure 2 is used. The dotted line in figure 2 indicates the additional part of the solution process implemented for improving lift prediction. The approach adopted in this study is to initially calculate the boundary layer solution with the inviscid wake geometry. The resulting edge velocity distribution on the airfoil is used to update the vorticity distribution and subsequently the streamfunction. This updated streamfunction is then 
used to determine the new wake geometry in the same way as. The calculations are repeated with the new wake geometry to update the lift. This approach significantly improves the lift prediction over a wide variety of cases as will be seen later. Figure 3 shows the wake geometry for DU 97-W-300 airfoil at $\alpha=8^{\circ}$. There is a noticeable deviation in the wake geometry for the improved method (denoted as viscous wake) compared to the existing method (denoted as inviscid wake). The wake computed using the new method (Figure 2) results in a reduced downwash when compared to the old method (Figure 1) and hence reducing the magnitude of lift coefficient. Figure 4(a) presents a case where lift discrepancy is observed in RFOIL predictions and the updated wake result in an improved prediction. The agreement with experimental results is significantly improved. Both the magnitude of lift coefficient and the lift slope are in better agreement when compared with the experimental data. ${ }^{5}$

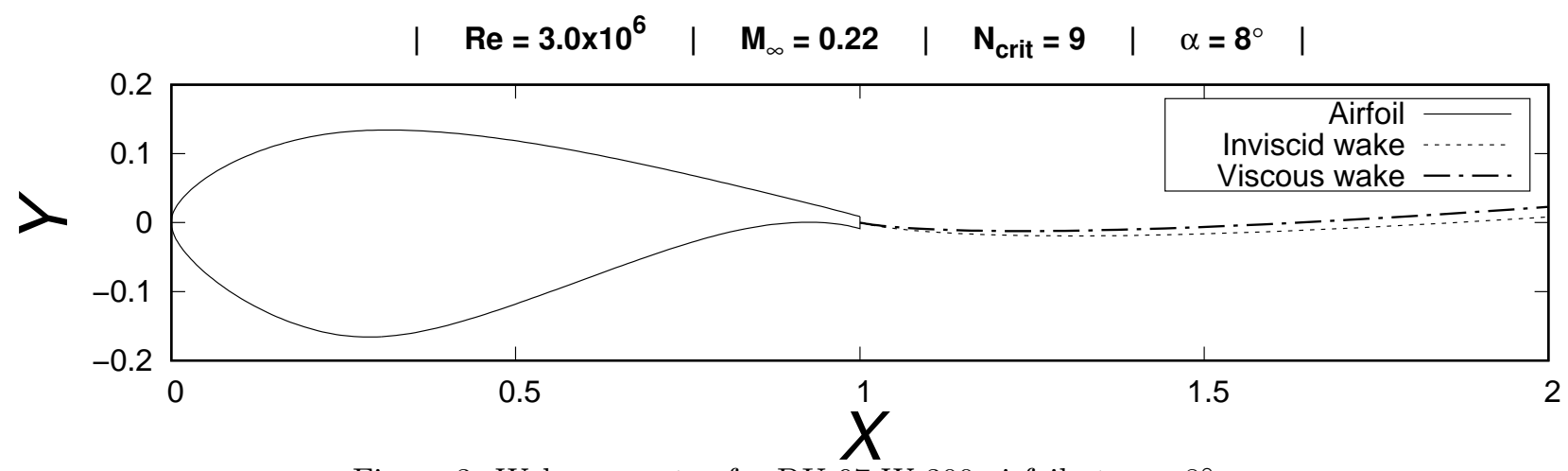

Figure 3: Wake geometry for DU 97-W-300 airfoil at $\alpha=8^{\circ}$

However, with the updated wake geometry, the drag prediction becomes inaccurate as seen in Figure 4(b). The drag prediction is reasonable at lower angles of attack as long as the drag does not rise significantly with increasing angle of attack. Comparing Figures 4(a) \& 4(b), we observe that the drag discrepancy occurs as soon as the lift curve becomes non-linear. Non-linearity of the lift curve indicates the onset of flow separation. A detailed analyses of the solution procedure reveals that as the flow approached separation, the shape factor is artificially restricted in order to avoid numerical difficulty. This is achieved by prescribing a shape factor value as soon as flow separation is approached. This prescribed shape factor in turn modifies the pressure gradient that the equations see, resulting in a smooth convergence. This approach is supported by the argument that in case of flow separation, the accuracy of the solution tends to decline anyways and a small modification would not significantly affect the result. However, when the wake geometry is updated with the modified vorticity distribution, this results in limiting the resulting shape factor below the value corresponding to flow separation. This artificial restriction makes the method behave as if the flow never separates. Although the lift follows the same behavior as before, the drag never rises post stall. Since this issue arises because of workaround to overcome the separation singularity problem, the drag is not updated when using the updated wake geometry. Figures $4(\mathrm{c}) \& 4(\mathrm{~d})$ present the drag curve and the lift drag polar with the corrected drag and improved lift formulation. It is also worth noting that since the solution procedure has been modified, the drag correction ${ }^{2}$ needs to be re-calibrated. Using the drag value from the first wake iteration also avoids this exercise. In Figure 4, the drag results have been presented for the complete angle of attack range evaluated for the simulations with RFOIL. As soon as the flow separates, the drag rises exponentially. Since the performance of the airfoil near the $(l / d)_{\max }$ point is more significant in assessing the quality of prediction of an airfoil design and analysis method like RFOIL, the drag results for the subsequent sections will be presented for a smaller range to magnify the region where flow separation sets in. The significance of this exercise will become clear in the discussion presented in further sections.

\section{Results}

In this section, the results for validating the improved lift prediction method are presented. The proposed method for improving lift prediction for wind turbine airfoils in RFOIL has been tested on a wide range of airfoils at various operational conditions. Figures 5-8 present a selection of test cases out of several that were used to validate the method. The test cases present several airfoils that are used in wind turbine applications at various sections of the blade; in-board, mid-board and out-board regions. A significant im- 


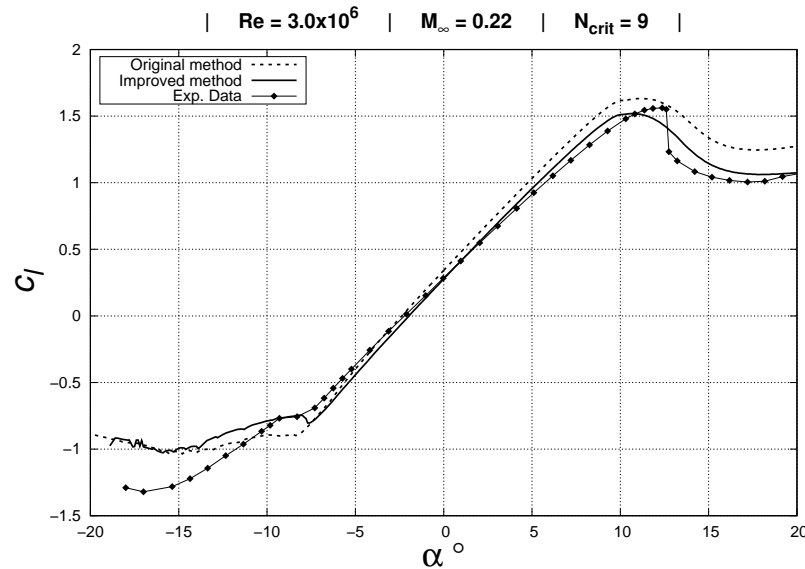

(a) Improved lift prediction with wake update approach

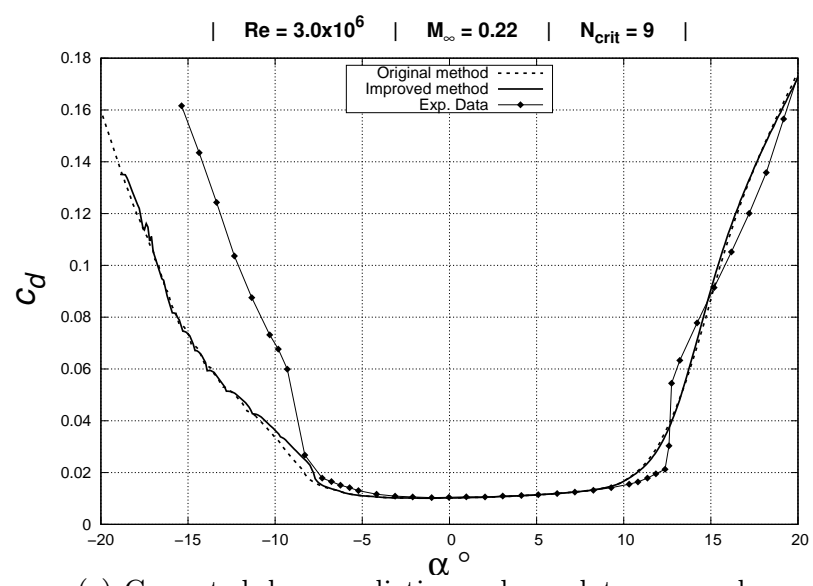

(c) Corrected drag prediction wake update approach

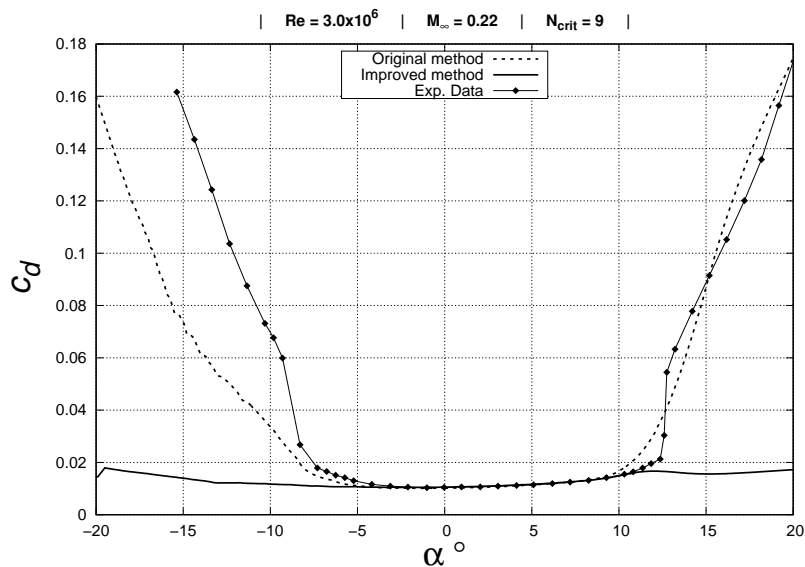

(b) Drag prediction discrepancy with wake update approach

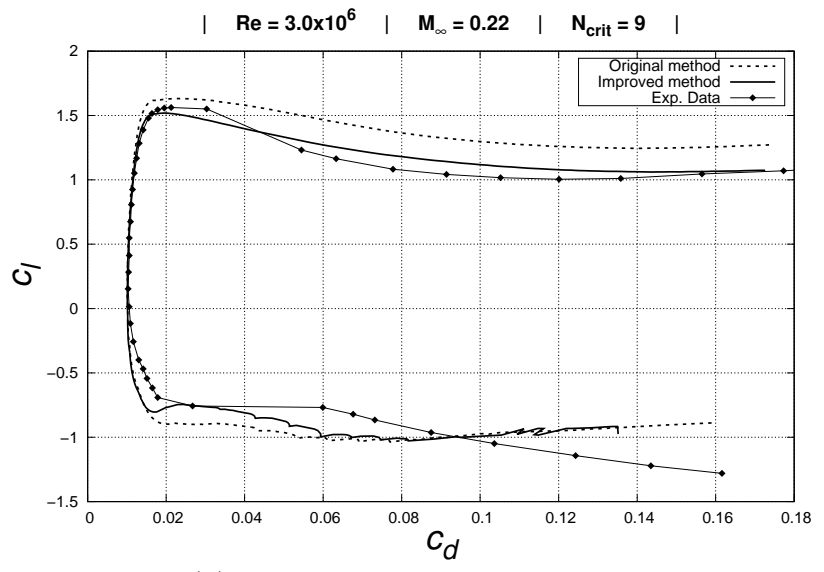

(d) Comparison of Lift Drag polar

Figure 4: DU 97-W-300 : Lift and drag prediction with and without wake geometry modification compared with experimental data ${ }^{5}$

provement was observed for all the test cases considered. The analysis presented in this section is computed for incompressible flow conditions $\left(M_{\infty}=0.00\right)$ for the conditions of natural transition with a freestream turbulence intensity of $0.07 \%\left(N_{\text {crit }}=9\right)$. The level of free-stream turbulence used in this study is within the range found in wind tunnels. Since experimental data in public domain is only available from certain wind tunnels, the corresponding levels of freestream turbulence are used. It is important to note that, in XFOIL like methods, the free-stream turbulence levels only affect the transition locations while the increased mixing in the turbulent boundary layer that leads to a delay of flow separation is not accounted for. ${ }^{6}$ Thus at elevated free-stream turbulence levels, the quality of prediction is expected to deteriorate anyway.

Even for thinner airfoils, there is a noticeable improvement in lift prediction as seen from the case of NACA $63_{3}-418$ airfoil in figure 5. For thicker airfoils, the over-prediction in lift becomes more severe. With the improved lift method, the lift prediction is in better agreement with the experimental data, especially in the linear lift range. A detailed observation of Figures 5(a) \& 5(b) indicates that with the improved method, the maximum lift is under-predicted and the airfoil stalls earlier when compared to the experimental data and the existing version of RFOIL. This observation is not completely true as can be seen in the results of drag coefficient in Figures 5(c) \& 5(d). The figures show the drag coefficient which is almost identical for both the improved and the original RFOIL method, indicating that the airfoil stalls at the same angle of attack in both methods. The experimental results for drag coefficient indicates that the stall occurs later than predicted by the computations. Onset of stall (flow separation) is indicated by the deviation of lift curve from its linear behavior and the sharp rise in drag coefficient. This discrepancy in predicting the onset of stall is observed in all the cases analyzed in this study. The discrepancy seems to lie in the closure relation 
for turbulent flow which regulate the onset of flow separation.

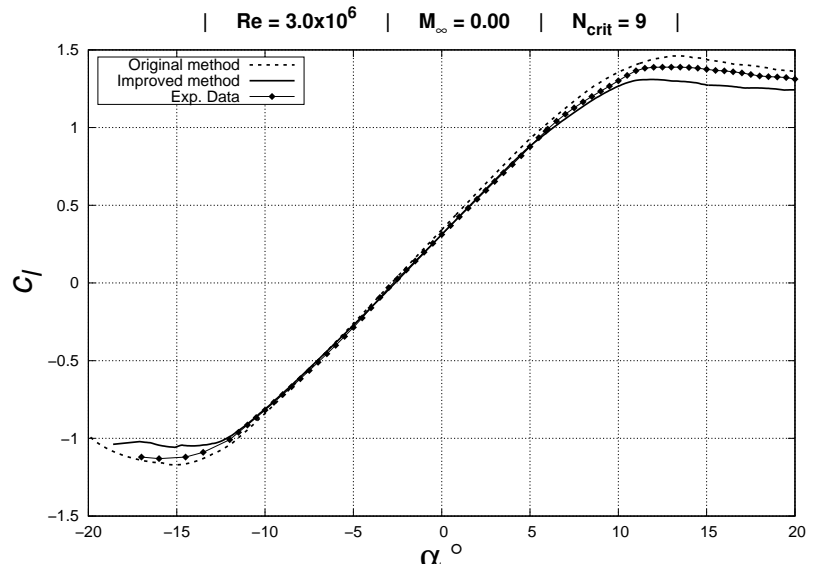

(a) Improved lift prediction with wake update approach

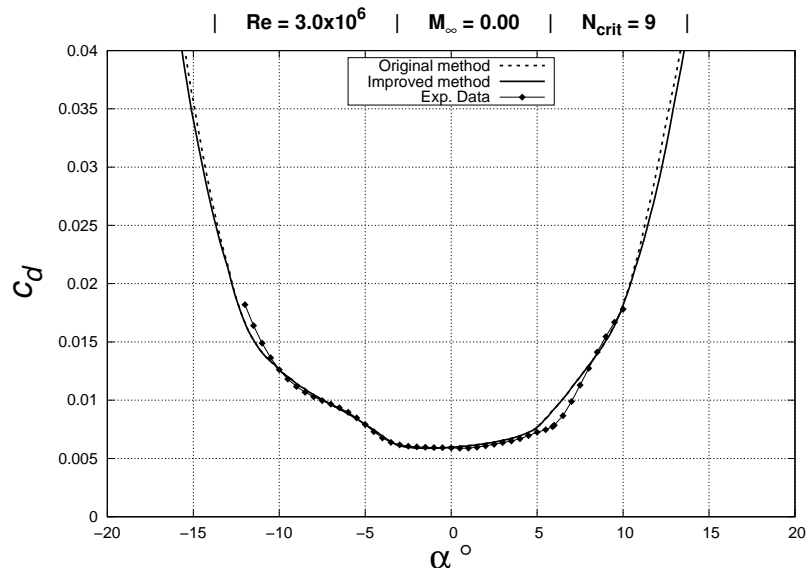

(c) Corrected drag prediction with wake update approach

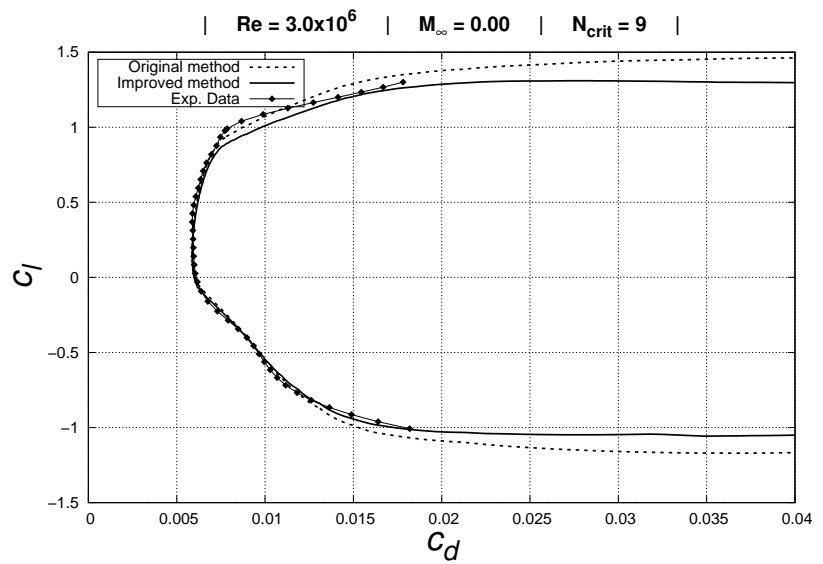

(e) Lift drag polar with wake update approach

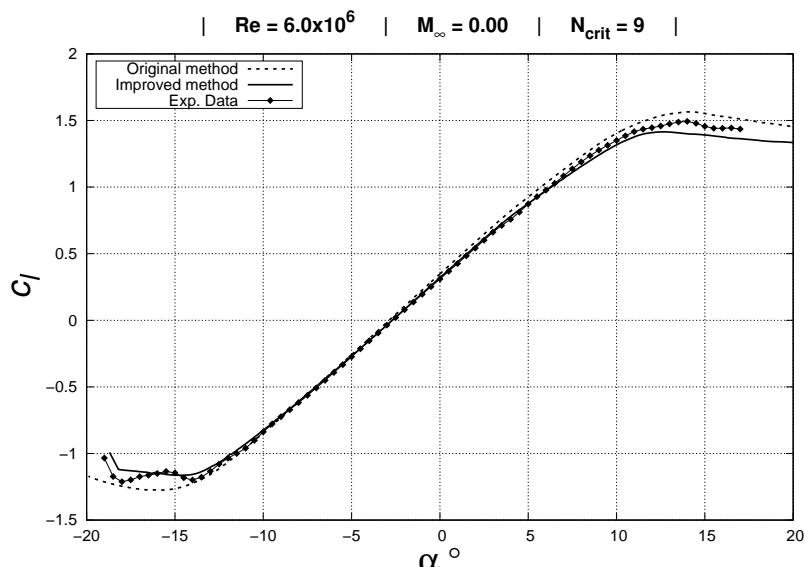

(b) Improved lift prediction with wake update approach

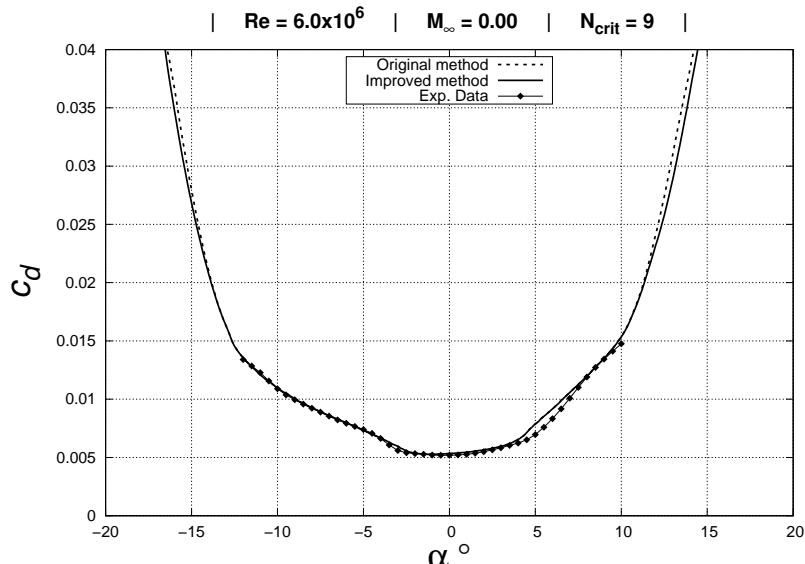

(d) Corrected drag prediction with wake update approach

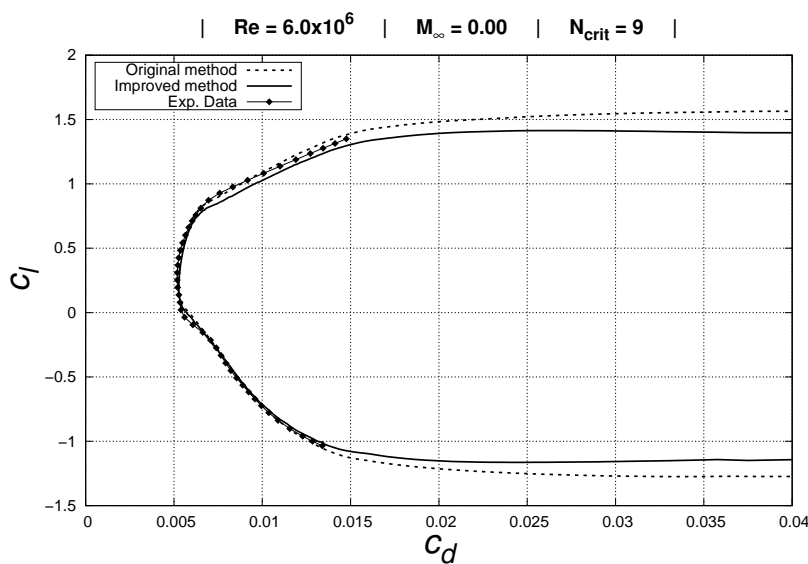

(f) Lift drag polar with wake update approach

Figure 5: NACA $63_{3}-418$ : Lift and drag prediction with and without wake geometry modification compared with experimental data ${ }^{7}$

Further research is needed to reformulate these closures in order to improve the prediction of maximum lift coefficient. This discrepancy was already existent in the original RFOIL method but was being masked by the lift over-prediction problem. The improved lift formulation only revealed an existing shortcoming of the existing RFOIL method. Similar behavior is seen in case of other test cases analyzed in this study. The maximum lift coefficient is under-predicted for AH 93-W-257, AH 93-W-300 and AH 94-W-301 airfoils for as seen in Figures 6, 7 \& 8. In Figures 7(b) \& 8(b), the lift over-prediction is not completely resolved. Hence, 
it appears that the maximum lift is not being under-predicted as observed in other cases.

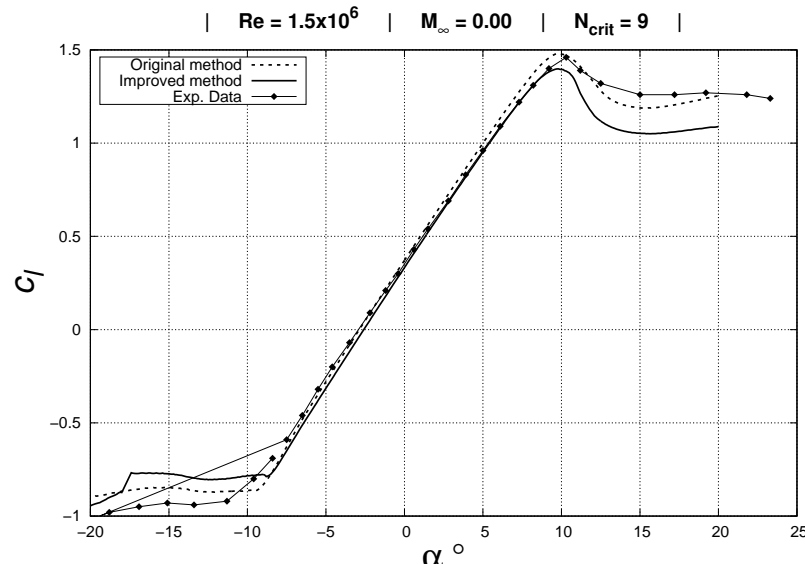

(a) Improved lift prediction with wake update approach

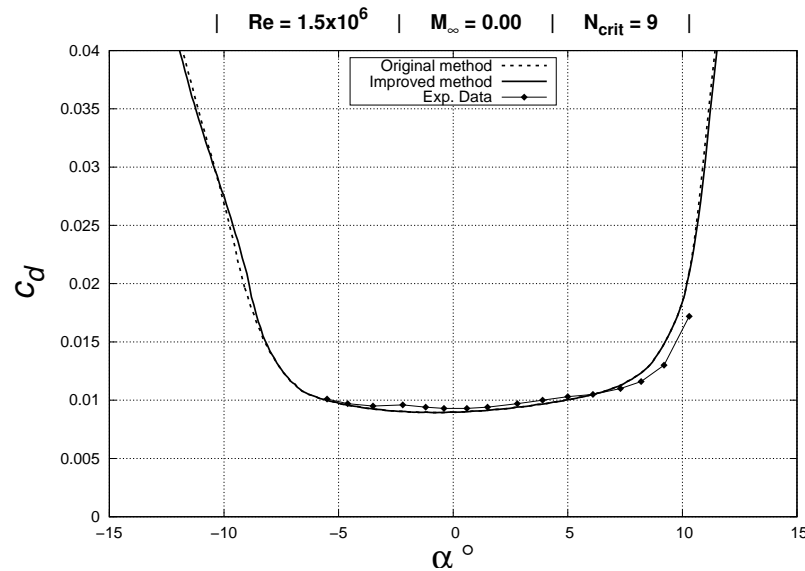

(c) Corrected drag prediction with wake update approach

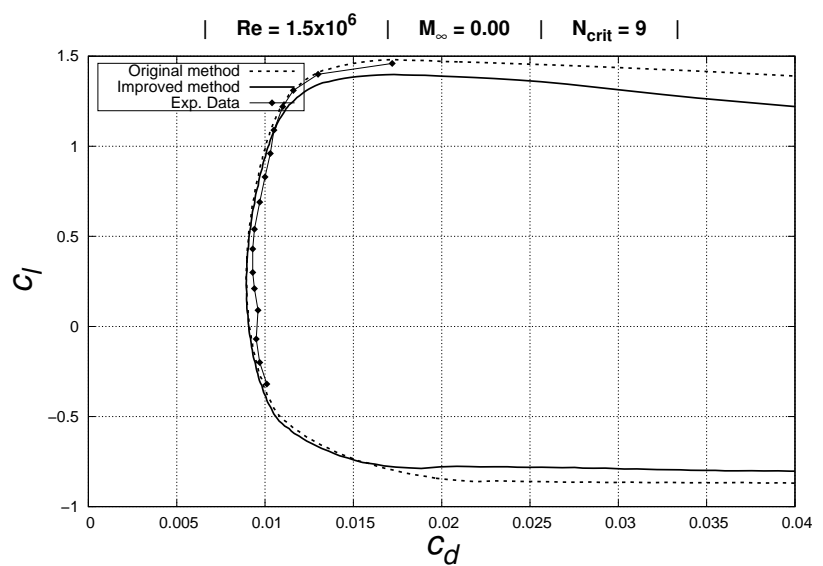

(e) Lift drag polar with wake update approach

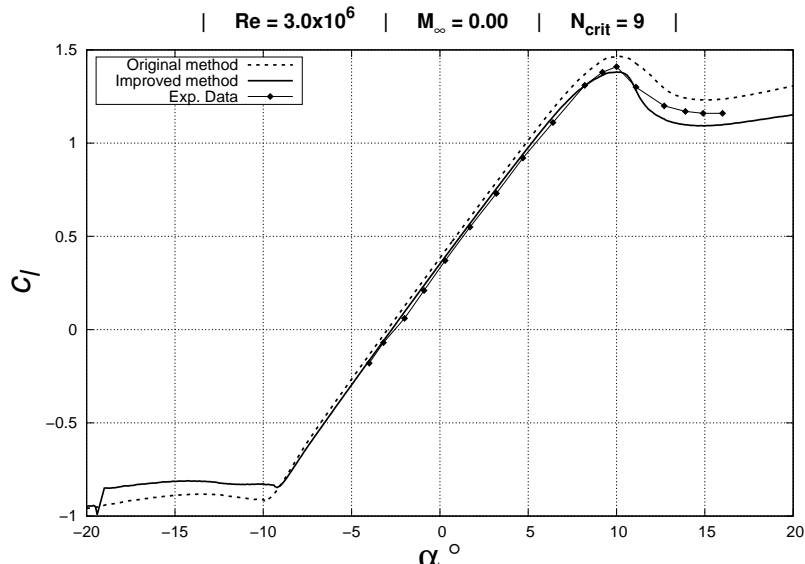

(b) Improved lift prediction with wake update approach

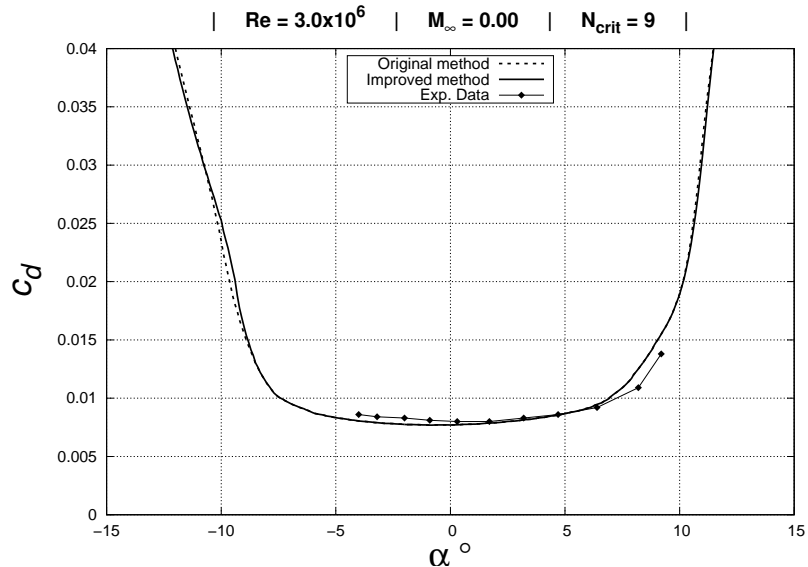

(d) Corrected drag prediction with wake update approach

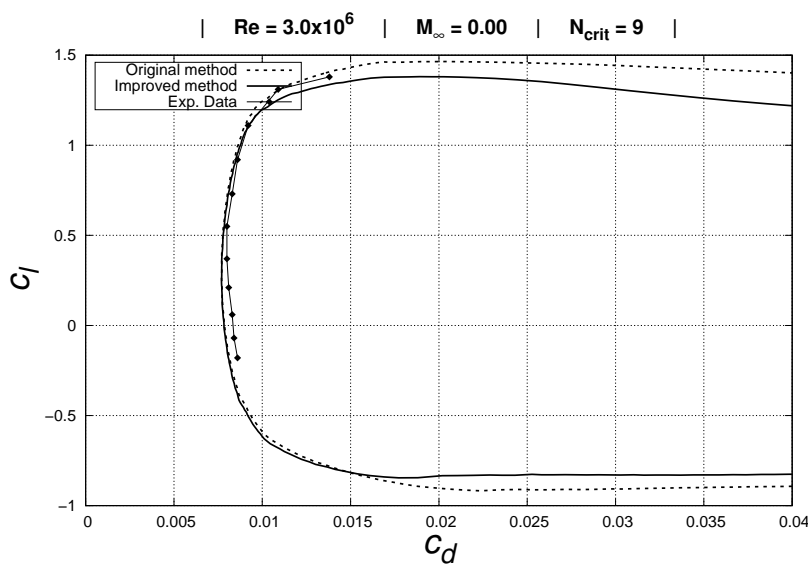

(f) Lift drag polar with wake update approach

Figure 6: AH 93-W-257 : Lift and drag prediction with and without wake geometry modification compared with experimental data ${ }^{8}$

The reason behind the discrepancy seems to be the trailing edge height $\left(h_{T E}\right)$ which is non zero for AH 93-E-257, AH 93-W-300 and AH 94-W-301 airfoils as shown in table 1. As the trailing edge height increases, so does the over-prediction of lift. This problem has two aspects to it. The lift over-prediction is partly caused by the wake geometry being derived from the inviscid solution which is improved with the improved method. The other aspect of error in lift is due to the trailing edge panel method formulation where a source strength is associated with the trailing edge panel. This aspect is discussed in Subsection (V.A). 


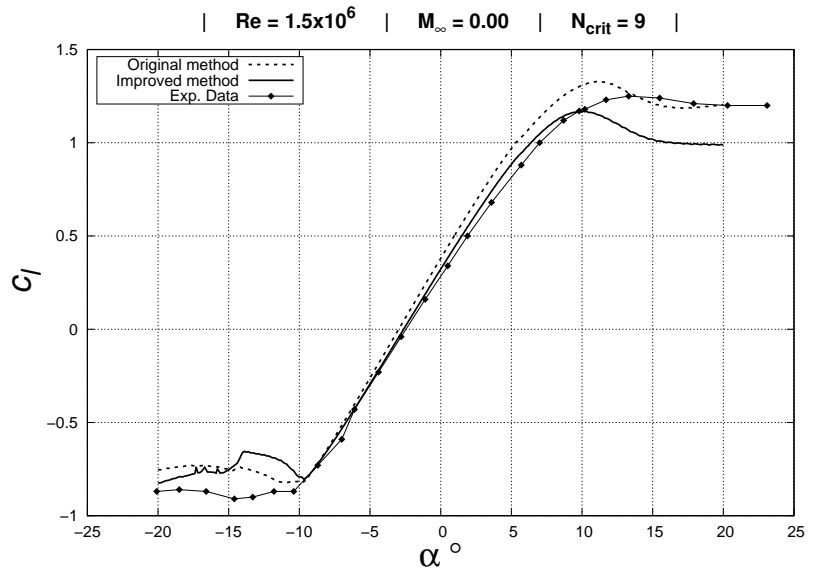

(a) Improved lift prediction with wake update approach

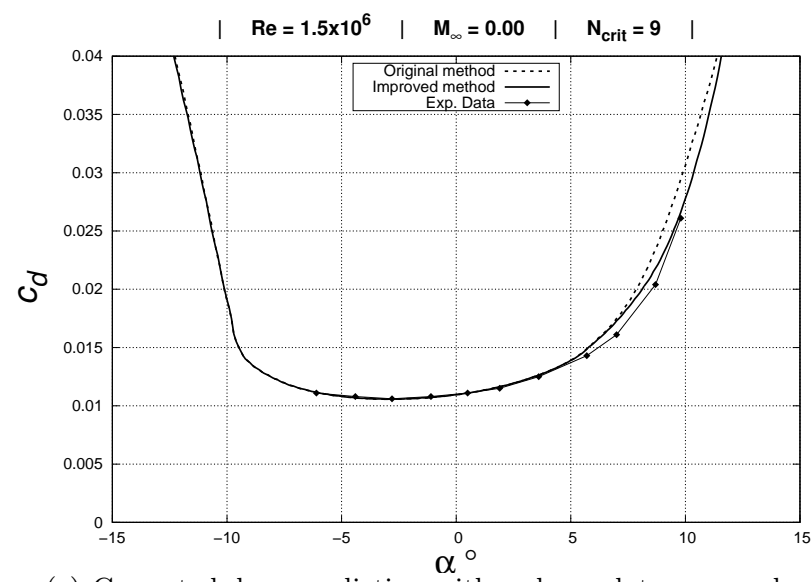

(c) Corrected drag prediction with wake update approach

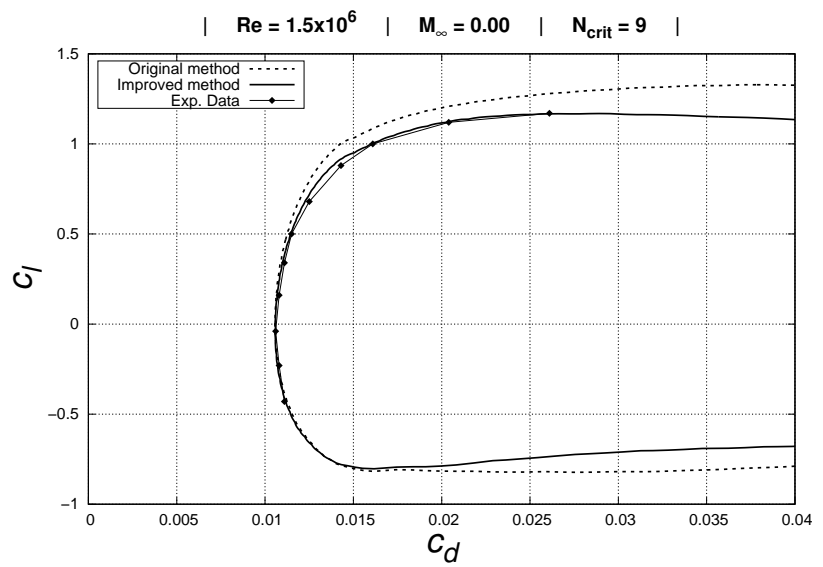

(e) Lift drag polar with wake update approach

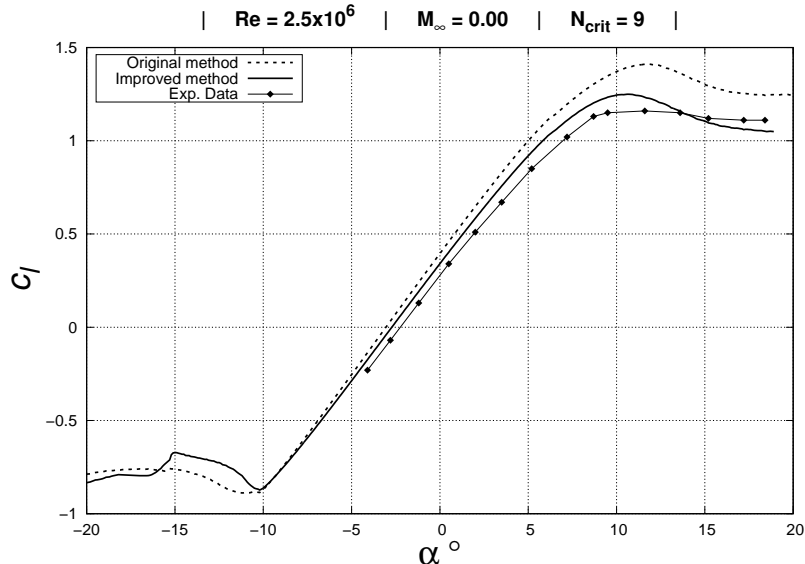

(b) Improved lift prediction with wake update approach

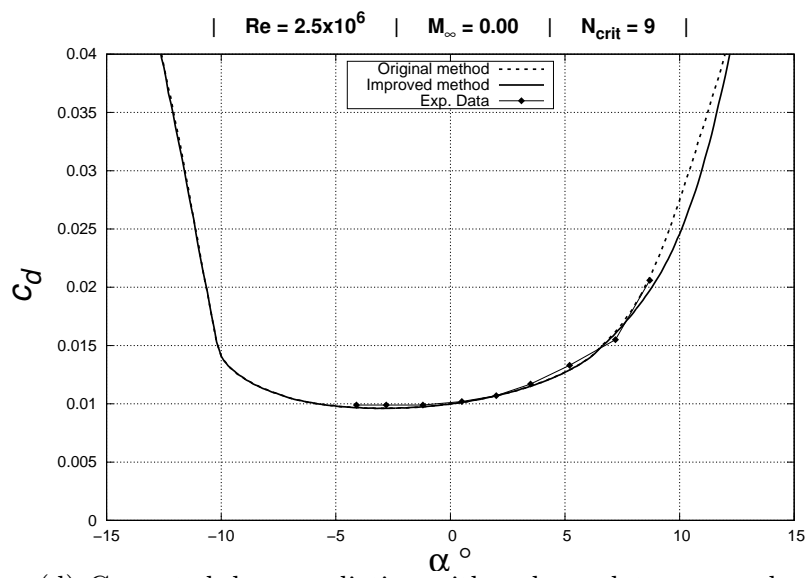

(d) Corrected drag prediction with wake update approach

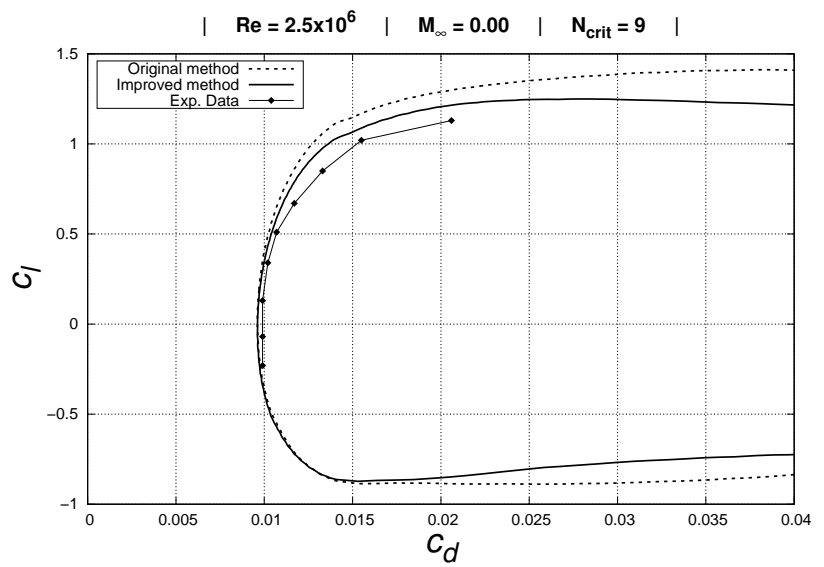

(f) Lift drag polar with wake update approach

Figure 7: AH 93-W-300 : Lift and drag prediction with and without wake geometry modification compared with experimental data ${ }^{8}$

A wide range of airfoils of thickness varying from $12 \%$ to $40 \%$ of chord length were tested in this study with the aim to test the improved lift prediction method. A noticeable improvement was observed in all cases. In order to avoid redundancy, only a few results have been presented in this work. The post stall lift and drag prediction are still not accurate with the present method as this type of a computational method for prediction flow around airfoils becomes inaccurate as the boundary layer becomes too thick when separation sets in. This leads a general inaccuracy in the results in the near and post stall region. In most of the 


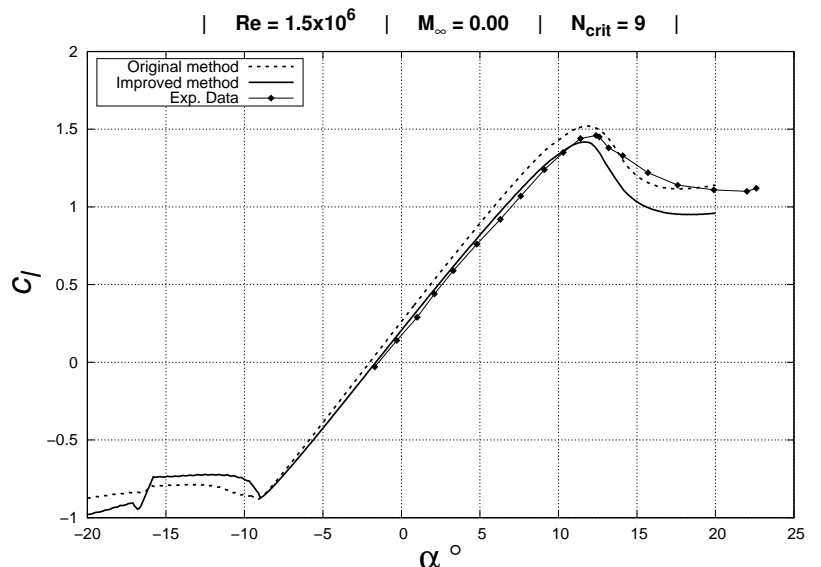

(a) Improved lift prediction with wake update approach

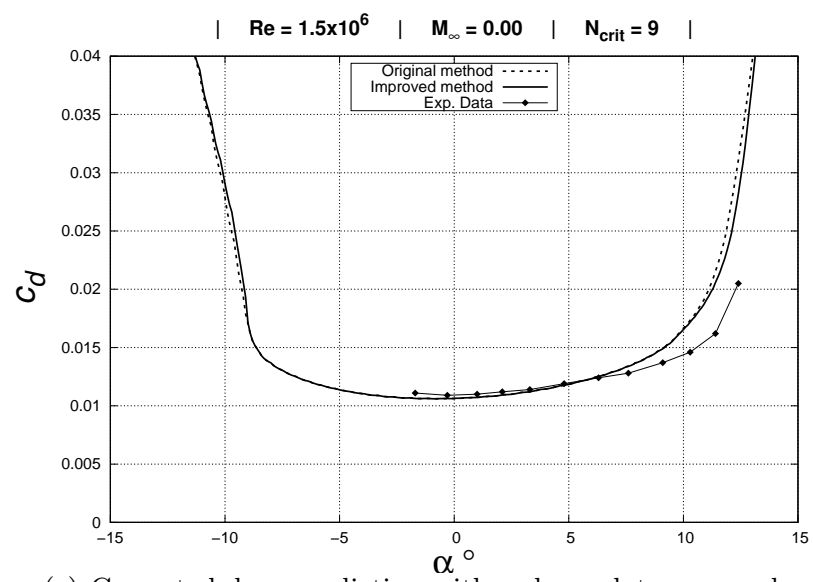

(c) Corrected drag prediction with wake update approach

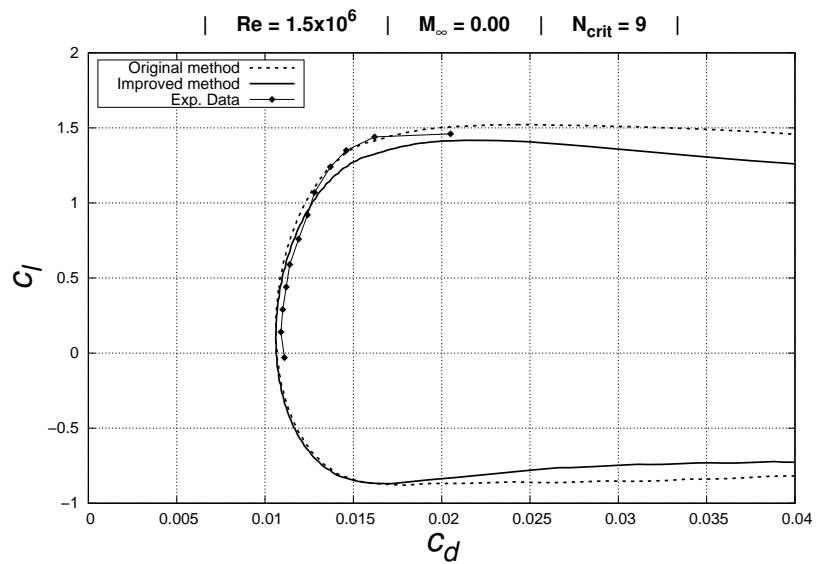

(e) Lift drag polar with wake update approach

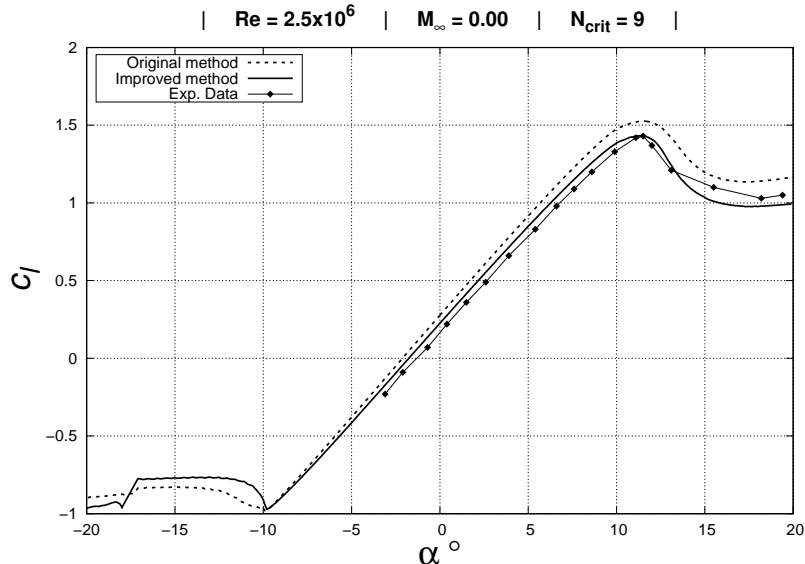

(b) Improved lift prediction with wake update approach

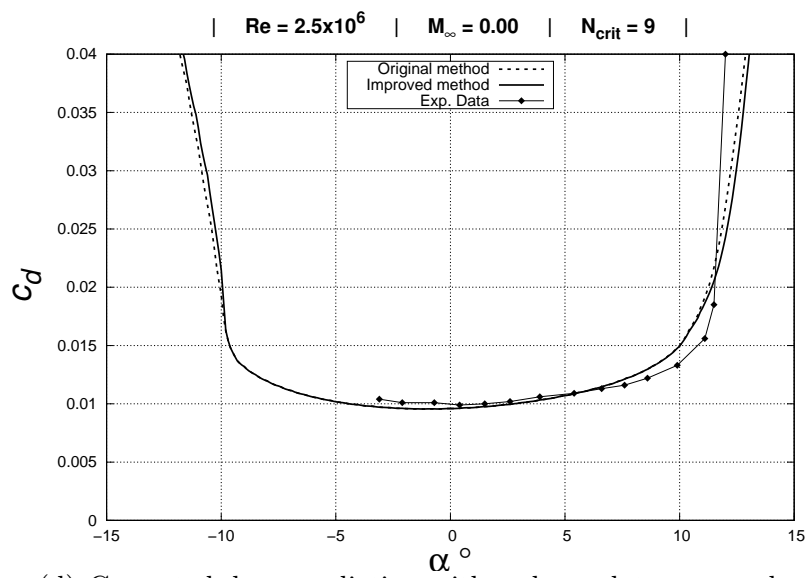

(d) Corrected drag prediction with wake update approach

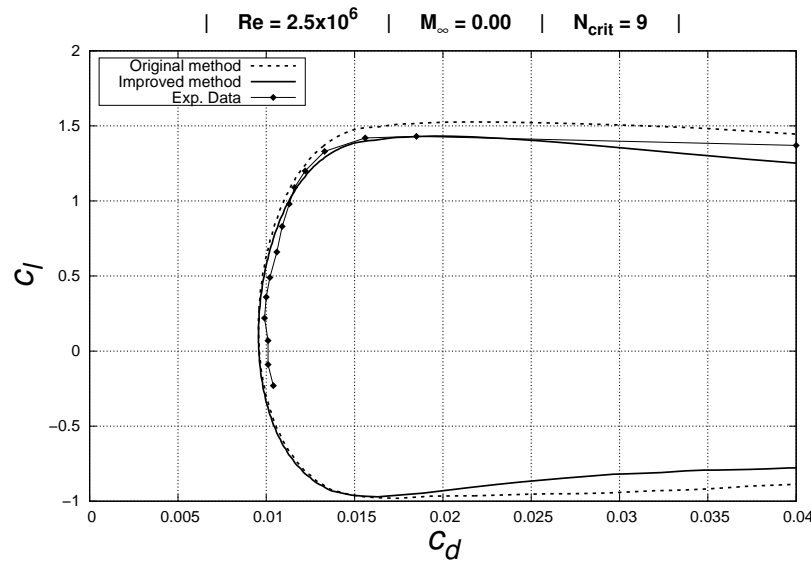

(f) Lift drag polar with wake update approach

Figure 8: AH 94-W-301 : Lift and drag prediction with and without wake geometry modification compared with experimental data ${ }^{8}$

cases analyzed in this study, the $(l / d)_{\max }$ is marginally under-predicted except for the cases where the lift over-prediction still exist due to the effect of thick trailing edge. This aspect is quite significant for airfoil design as a conservative prediction of airfoil performance $\left((l / d)_{\max }\right)$ would lead to a more reliable design.

\section{Other Observations}


Through the course of this study, several observations were made regarding the scope and accuracy of the prediction in RFOIL and other such IBL methods. In context to lift prediction, two key issues were identified. These were the prediction accuracy for the case of thick trailing edge airfoils and the influence of elevated freestream turbulence levels on airfoil performance. The following subsections present the observations and a general discussion on the observed results in order to explore possibilities for further improvements.

\section{V.A. Trailing edge formulation in the inviscid calculation}

Several researchers have reported the under-prediction in drag observed with XFOIL like methods for TTE airfoils. ${ }^{9}$ It is interesting to note that a significant over-prediction in lift also exists in this case and the lift slope and the maximum lift are also over-predicted for such airfoils. This discrepancy in lift is not fully resolved by using the wake update method as seen in the previous results and in figure 9. When the trailing edge height becomes significant with respect to the chord length, the lift prediction with the updated wake is still inaccurate. The problem seems to arise from the trailing edge model present in RFOIL (and XFOIL). The trailing edge model mentioned here is not the blunt trailing edge boundary layer formulation proposed by Drela ${ }^{10}$ but the trailing edge panel definition in the inviscid formulation that seems to give rise to the discrepancy.

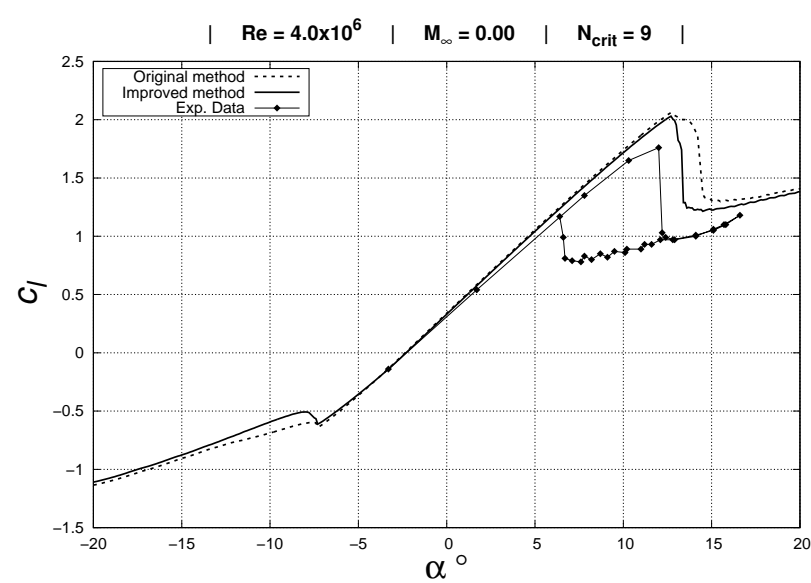

(a) Improved lift prediction with wake update approach

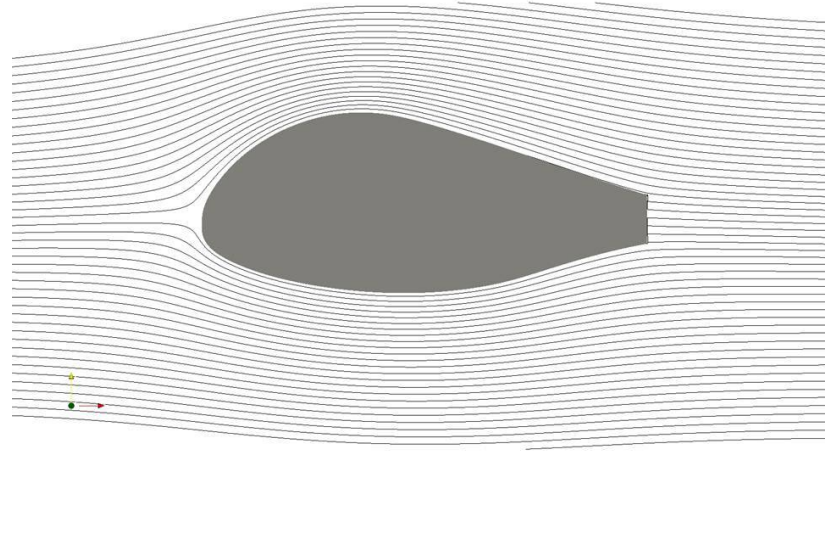

(b) Streamlines in RFOIL $\left(\alpha=0^{\circ}\right)$

Figure 9: Discrepancies in lift prediction in RFOIL for TTE airfoil (FX 77-W-400)

The trailing edge (base) panel has a source strength associated with it which results in a jet-like behavior in TTE airfoils where mass is constantly ejected from the base panel as seen in the streamline plot shown in figure $9(\mathrm{~b})$. This results in a reduction in base pressure, thereby affecting the pressure distribution on the airfoil surface as well as the wake. The source strength of the trailing edge panel has a significant effect on the wake geometry. Thus, updating the wake geometry has a marginal effect compared to the influence of the source strength of the trailing edge panel. Thus, only a small change in lift is observed with the improved method. In order to improve the lift prediction for TTE airfoils, the trailing edge formulation and the Kutta condition need to be reconsidered. The flow must separate at the upper and lower trailing edge points, which is accomplished by having a source strength across the trailing edge panel. This however does not accurately model the base pressure, thereby causing a discrepancy in the lift prediction. Further research is needed in this area to improve the panel method formulation for TTE airfoils.

\section{V.B. Effects of freestream turbulence on airfoil performance}

The performance of an airfoil is influence by several parameters describing the operating conditions. In methods like RFOIL, a wide variety of these parameters are included in the mathematical model. The 
most important operating parameters that affect the predicted airfoil performance in RFOIL are Reynolds number $(R e)$, Mach number $\left(M_{\infty}\right)$, angle of attack $(\alpha)$ and critical amplification factor $\left(N_{\text {crit }}\right)$. The critical amplification factor determines when laminar to turbulent transition happens and it is dependent on the freestream turbulence level according to Mack's relation. ${ }^{11}$

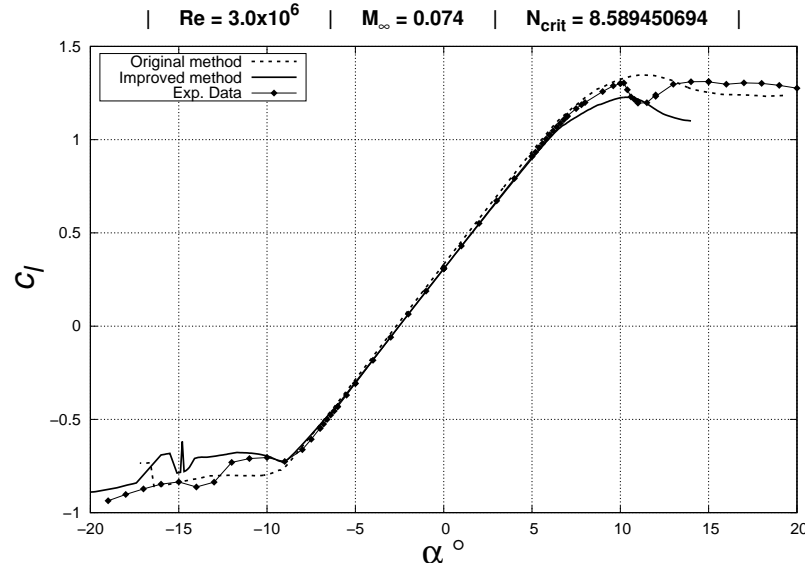

(a) Improved lift prediction with wake update approach

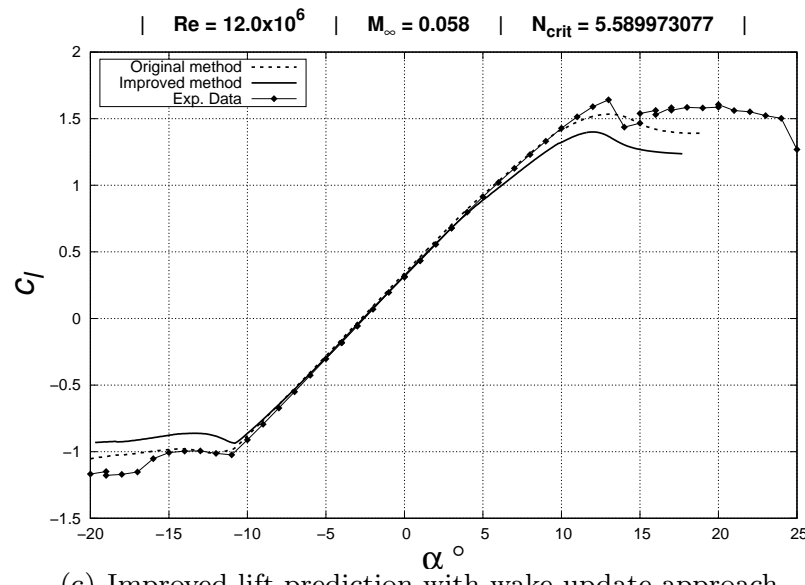

(c) Improved lift prediction with wake update approach

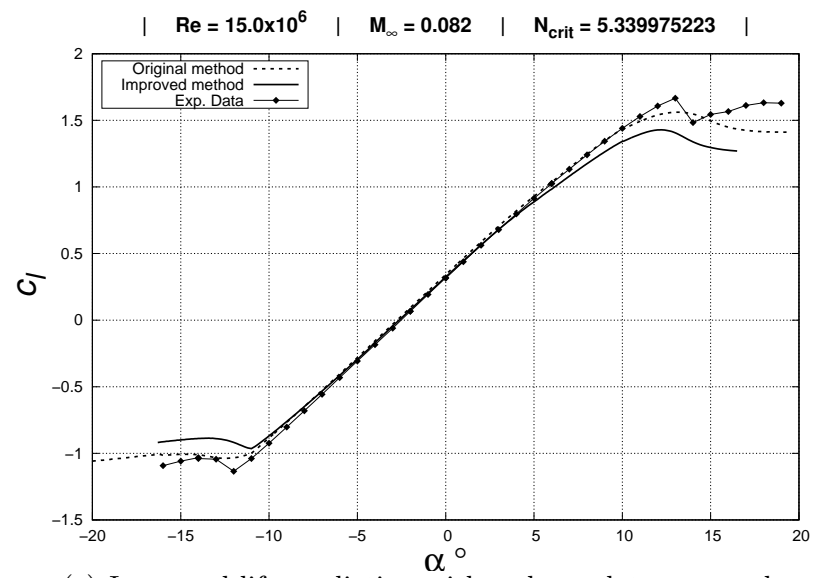

(e) Improved lift prediction with wake update approach

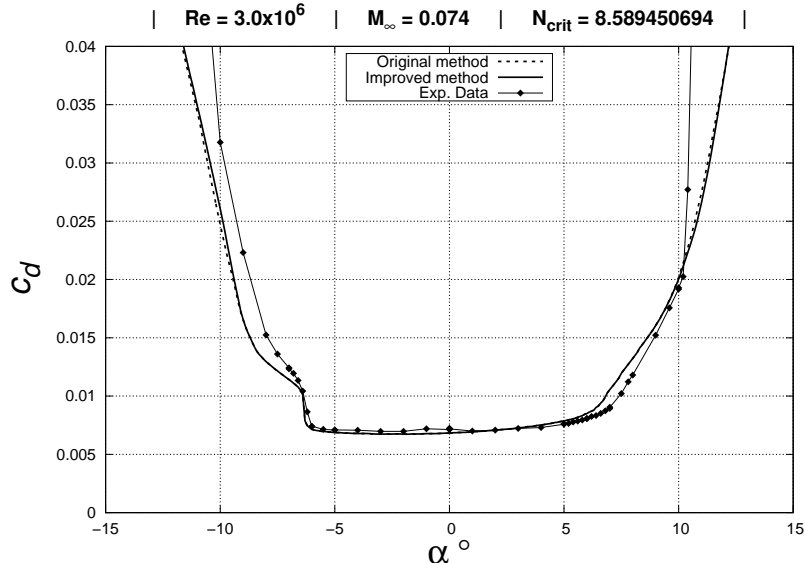

(b) Improved lift prediction with wake update approach

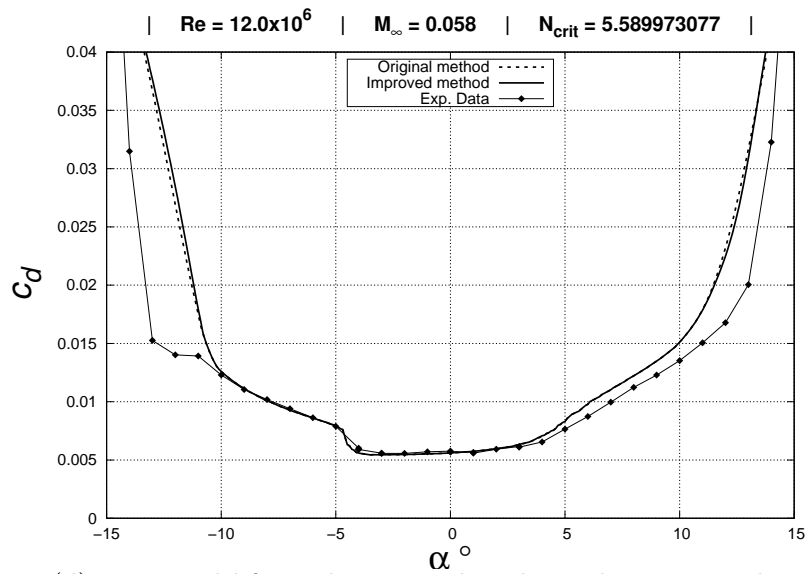

(d) Improved lift prediction with wake update approach

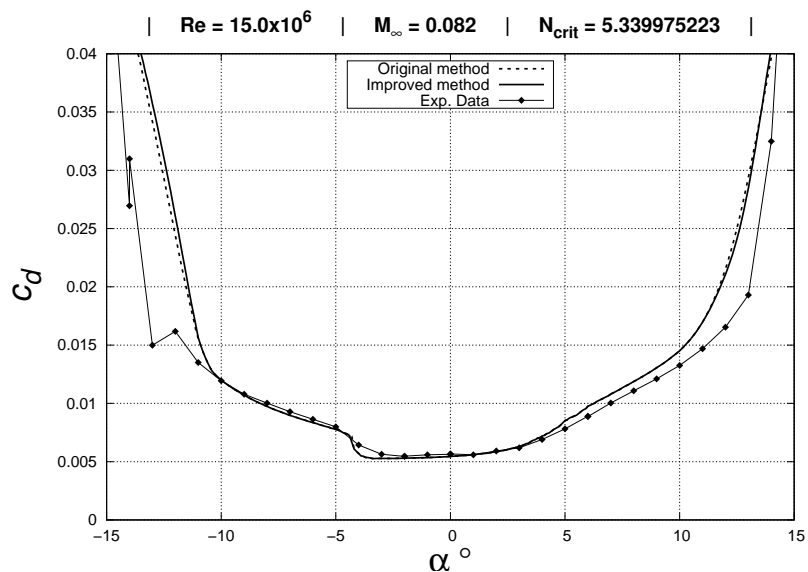

(f) Improved lift prediction with wake update approach

Figure 10: DU 00-W-212 : Lift and drag prediction with and without wake geometry modification compared with experimental data ${ }^{12}$

The value of $N_{\text {crit }}$ decreases with increasing freestream turbulence intensity thus causing an early onset of laminar to turbulent flow transition. Despite early flow transition, airfoils operating at elevated turbulence levels have a higher maximum left due to delayed onset of stall. ${ }^{6}$ This is contrary to what is expected 
when the flow transitions earlier. Figure 10 shows the predicted aerodynamic characteristics of DU 00-W212 airfoil at various Reynolds numbers and freestream turbulence levels using RFOIL in comparison with experimental data. ${ }^{12}$ The wind tunnel tests were carried out in a pressurized wind tunnel in order to reach high Reynolds numbers. As seen in the previous sections, the lift coefficient in over-predicted in RFOIL calculations. But Figure 10(a) shows no such over-prediction at a Reynolds nuber of $3 \times 10^{6}$. The improved lift method stall earlier and under-predicts the maximum lift. For higher Reynolds number, the maximum lift is under-predicted even with the existing RFOIL method as seen in Figures 10(c) \& 10(e). The drag curves (Figure 10(b), 10(d) \& 10(f)) show that the angle of attack corresponding to the onset of flow separation is higher for the experimental results than for RFOIL predictions. This is the same behavior observed in the test cases mentioned analyzed in the previous sections. The reason behind the higher maximum lift observed in the experimental data is the elevated freestream turbulence levels observed in these cases. RFOIL predictions are reasonable around an $N_{\text {crit }}$ value of 9 which corresponds to a freestream turbulence level of $0.07 \%$ which is typically found in wind tunnels. Higher freestream turbulence level promotes greater mixing in the boundary layer which delays flow separation. However, this phenomenon is not modelled in RFOIL as the freestream turbulence level has no effect on the development of the turbulent boundary layer. The effects of freestream turbulence level on integral boundary layer has been modelled by Evans et. al., ${ }^{13}$ which can be used to improve the prediction accuracy for these cases in RFOIL.

\section{Conclusions}

To enable accurate prediction of lift for thick wind turbine airfoils, a modification to the existing lift calculation method in integral boundary layer methods like RFOIL (or XFOIL) has been developed. The proposed correction improves the prediction of lift for thick airfoils as long as the trailing edge height is small. It is also observed that the RFOIL method predicts an early onset of flow separation in comparison to experimental data. The results of the improved lift prediction method are in good agreement with experimental data in the linear lift range and improving the prediction for the onset of flow separation will improve the agreement in near and post stall region as well. Further observations were made on the lift prediction of TTE airfoils where the lift is over-predicted in RFOIL calculations even with the improved method. The trailing edge panel formulation was identified as the cause for this behavior and further research is needed to evaluate this aspect. Finally, some observations are made on the effect of free-stream turbulence intensity on airfoil performance and the lack of accounting for it in RFOIL which leads to a discrepancy in lift prediction.

\section{References}

\footnotetext{
${ }^{1}$ Rooij, R. P. J. O. M. Van, "Modification of the boundary layer calculation in RFOIL for an improved stall prediction," Tech. Rep. IW-96087R, TU Delft, 1996.

${ }^{2}$ Ramanujam, G., Özdemir, H., and Hoeijmakers, H., "Improving Airfoil Drag Prediction," 34th Wind Energy Symposium, No. 0748, AIAA SciTech, January 2016.

$>3$ Drela, M., "XFOIL: An analysis and design system for low Reynolds number airfoils," Low Reynolds number Aerodynamics, Springer, Heidelberg, Berlin, 1989, pp. 1-12.

${ }^{4}$ Drela, M. and Youngren, H., XFOIL 6.9 User Primer, Massachusetts Institute of Technology, Cambridge, MA 021394307, 2001.

${ }^{5}$ Timmer, W. A., "Ontwerp en windtunneltest van profiel DU 97-W-300," Tech. Rep. IW-98003R, TU Delft, 1998, in Dutch.

${ }^{6}$ Ramanujam, G., Improving aerodynamic prediction methods for wind turbine airfoils, Master's thesis, University of Twente, August 2015, Research group : Engineering Fluid Dynamics, Report Number : 207.

${ }^{7}$ Abbott, H. and von Doenhoff, A. E., "Measurements NACA in LTT-Langley," Tech. Rep. 422, NACA, 1949.

${ }^{8}$ Althaus, D., "Stuttgarter Profilkatalog II: Niedriggeschwindigkeitsprofile," University of Stuttgart, 1996.

$\checkmark{ }^{9}$ Grasso, F., "Modeling and Effects of Base Drag on Thick Airfoils Design," 32nd ASME Wind Energy Symposium, No. 0168, AIAA SciTech, 2014.

${ }^{10}$ Drela, M., "Integral boundary layer formulation for blunt trailing edges," 7th Applied Aerodynamics Conference, Fluid Dynamics and Co-located Conferences, No. 89-2166-CP, AIAA, 1989.

${ }^{11}$ Mack, L., "Transition prediction and linear stability theory," In AGARD Laminar-Turbulent Transition, Vol. 1, 1977, pp. 1.1-1.22.

${ }^{12}$ Ceyhan-Yilmaz, Ö., Pires, O., Manolesos, M., and C.Simao-Ferreira, "Blind test campaign," Tech. Rep. D2.10, AVATAR project report, 2015, WP2, Task 2.3 .

${ }^{13}$ Evans, R. L. and Horlock, J., "Calculation of the Development of Turbulent Boundary Layers With a Turbulent Freestream," Journal of Fluids Engineering, Vol. 96, No. 4, 1974, pp. 348-352.
} 